\title{
MICROFÚTBOL EN EL BARRIO ARBORIZADORA ALTA: ENTRE LO RECREATIVO, LO DEPORTIVO, LA VIOLENCIA Y LA CONVIVENCIA
}

\author{
SOCCER SMALL-SIDED GAMES AT THE ARBORIZADORA ALTA NEIGHBORHOOD: \\ BETWEEN RECREATION, SPORTS, VIOLENCE AND COEXISTENCE
}

\author{
MICROFUTEBOL NO BAIRRO ARBORIZADORA ALTA: ENTRE \\ RECREAÇÃO, ESPORTES, VIOLÊNCIA E CONVIVÊNCIA
}

Hamilton Hilarion Montero ${ }^{1}$

Resumen

Este artículo se realizó a partir de una propuesta etnográfica ejecutada en el barrio Arborizadora Alta, con el fin de reconocer y reflexionar en torno a las dinámicas que se dan socialmente dentro del contexto del juego del microfútbol. La investigación sugiere que al institucionalizarse el microfútbol, es decir, hacerlo un deporte, este llegará a generar más problemas de convivencia que soluciones, ya que aumenta la violencia por múltiples factores tanto internos como externos. Sin embargo, también sugiere que al no institucionalizarse y permitirse que el juego se desarrolle en ámbitos más informales, podría tender menos a la violencia y más a la otredad.

Palabras clave: juego; violencia; recreación; deporte; convivencia

This article is the product of an ethnographic proposal carried out in the Arborizadora Alta neighborhood, in order to recognize and reflect around the social dynamics given within the context of soccer small-sided games. Research suggests that by institutionalizing soccer small-sided games, that is, by making it a sport, it will generate more coexistence conflicts than solutions, since it increases violence by multiple factors, both internally and externally. However, it also suggests that by not institutionalizing the game, and allowing it to develop in more informal settings, it would incline less towards violence and more towards otherness.

Keywords: game; violence; recreation; sport; coexistence

1 Licenciado en Recreación y Turismo, Universidad Pedagógica Nacional. Correo electrónico: fef_hhilarionm342@pedagogica.edu.co. 


\section{Resumo}

Este artigo de pesquisa foi realizado a partir de uma proposta etnográfica realizada no bairro Arborizadora Alta, com o objetivo de reconhecer e refletir sobre as dinâmicas que ocorrem socialmente no contexto do jogo de microfutebol. A pesquisa sugere que, quando o microfoot é institucionalizado, ou seja, fazê-lo um esporte, isso poderia gerar mais problemas de convivência do que soluções, já que a violência aumenta devido a múltiplos fatores internos e externos. No entanto, também sugere que, ao não institucionalizar e permitir que o jogo se desenvolva em ambientes mais informais, poderia tender menos para a violência e mais para a alteridade.

Palavras chave: jogo; violência; recreação; esporte; vivendo juntos

Fecha de recepción: 3 de mayo de 2018

Fecha de evaluación: 24 de noviembre de 2018

Para citar este artículo:

Montero, H. (2018). Microfútbol en el barrio Arborizadora Alta: entre lo recreativo, lo deportivo, la violencia y la convivencia. Lúdica Pedagógica, 28, 99-106. 


\section{INTRODUCCIÓN}

En este artículo se hace un acercamiento desde el microfútbol como mediación/acción recreativa y recreo-deportiva, en cuanto a su función valorada desde el juego. Este último se aborda desde las manifestaciones recreativas, como constructor y fortalecedor del tejido sociocultural, esto es, se exploran las dinámicas que se desarrollan en torno a esta actividad en algunas canchas (campos de juego) del barrio Arborizadora Alta.

Huizinga (1972) propone y define el juego, entre otras cosas, como una actividad libre que conlleva

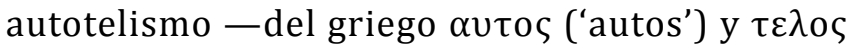
('telos') y que se define como acción con fin en sí misma-, reglas determinadas y aceptadas por sus participantes, una improductividad inherente, así como la posibilidad de ver el mundo desde una perspectiva diferente al propiciarse otras formas de ser. Dicho concepto, observado desde la línea de Manifestaciones Recreativas de la Licenciatura en Recreación y Turismo de la Universidad Pedagógica Nacional (UPN) podría considerarse una manifestación recreativa, ya que esta línea define y asume la recreación como la construcción de espacios y procesos de agenciamiento de otros mundos posibles es decir, de manera similar a como sucede con el juego.

En la UPN se entiende por recreación: "una mediación creativa que tiene por propósito aportar a la construcción del tejido sociocultural potenciando la imaginación y los lenguajes lúdicos [...]" (,UPN, 2016, p. 36). Esto nos lleva a pensar el juego, entonces, como una mediación, es decir, el no control de medios y fines sino el agenciamiento $^{2}$ de variables emergentes (que tienen cabida en la medida en que el juego transcurre), lo que permite la construcción de tejido sociocultural, y que es, por demás, el fortalecimiento de la convivencia, la tolerancia, las formas de ser, sentir y vivir, por parte de una comunidad.

Por lo anterior, este artículo tiene por objetivo mostrar todas las dinámicas que se dan en torno al microfútbol desde una perspectiva social en todo lo relacionado con la violencia, la convivencia y la función de la recreación y el deporte en ellas.

2 Palabra del latín agere, ago, agis que se utiliza para proponer espacios críticos no hegemónicos, que desafían lo normativo, lo impuesto y lo fijo a determinados agentes.

\section{CONTEXTUALIZACIÓN DEL} SECTOR Y DEL ESCENARIO

Arborizadora Alta es parte de la localidad de Ciudad Bolívar (localidad 19, UPZ 70), ubicada en el sur de Bogotá y que cuenta con 252 barrios, según reporte de la Alcaldía local del sector. Dicha localidad tiene un alto índice de conflictos (sociales, políticos, económicos, etc.), estigmatización e invisibilización por parte del Estado y la sociedad, que se reflejan en sus situaciones precarias, las cosas que de allí se dicen, así como en un gran cúmulo de culturas producto del desplazamiento forzado derivado del conflicto armado que tiene Colombia. Las incidencias más grandes en el sector son la violencia, la pobreza, la falta de educación, la alta natalidad y, en muchos casos, la ausencia de una vida digna, como lo mencionan Rodríguez y Moreno (2013):

Ciudad Bolívar se ha constituido en un escenario en el que como consecuencia de la expulsión masiva de personas de las distintas regiones del territorio colombiano, a causa del conflicto armado y la ejecución de mega proyectos, no sólo ha aumentado considerablemente su población, sino que además se matiza con las innumerables cargas semánticas asociadas con la exclusión, la pobreza, la inseguridad y, por supuesto, los remanentes de los conflictos sociales derivados del desplazamiento forzado. (P. 58).

Esta es la localidad que tiene el mayor número de desplazados en (26\%) y aunque la mayoría de sus habitantes son de los estratos más bajos, muchos de ellos realmente viven en la miseria o también llamada pobreza extrema. Entre otros factores se pueden destacar la presencia de grupos guerrilleros y paramilitares debido a la extensa área rural de dicha localidad, así como el peligro que representan los parques de juego, a los cuales resulta intimidante para la comunidad ir debido a la gran presencia de pandillas (Universidad del Rosario, s. f.).

Las dinámicas y visualizaciones de los resultados presentes en este artículo no se presentan desde una perspectiva lejana y de imaginarios, sino desde la autoría de un habitante del sector de hace más de 18 años, por lo que es un miembro conocido del lugar y no actúa ni hace las veces de extranjero en el lugar (por lo cual se asegura que no hay cambio de comportamientos por parte de los actores implicados 
y observados directamente). Precisamente esta es una de las razones que explica el interés de este artículo: el autor ha sido partícipe y observador propio del juego de microfútbol del lugar y de los diferentes campos de juego donde se adelantó la indagación, tanto en torneos como en no-torneos ${ }^{3}$, así como víctima de los factores de violencia, inseguridad $y$, en ocasiones, baja calidad de vida, entre otros que se dan allí. Esto ha dado lugar a unas vivencias y experiencias que permiten hacer un acercamiento desde el interior mismo de las situaciones presentadas en el sector desde hace muchos años (sin descartar que pudiera haber sesgo debido a la cercanía fraternal con el lugar).

\section{METODOLOGÍA}

Se direccionó entonces este artículo por una línea de investigación cualitativa, la cual según Fetterman (1989) tiene como características entender fenómenos y relaciones intrínsecas dadas, así como considerar datos según el contexto en que fueron obtenidos. Se eligió el método etnográfico, más específicamente el de tipología transversal, en donde se realiza la investigación de momentos determinados y se brinda la posibilidad de ver el mundo a través de la óptica de los actores observados (Boyle, 1994, citado en Murillo y Martínez-Garrido, 2010), teniendo en cuenta que también el autor del artículo expresa sus puntos de vista y experiencias adquiridas dentro del contexto.

La aproximación y la construcción de los resultados encontrados se hizo a partir de una serie de visitas a los dos parques más concurridos del sector, donde se realizan los torneos más grandes de microfútbol - según la perspectiva del autor y de algunos de sus amigos también habitantes de hace años del sector-. A partir de estas visitas se utilizó el diario de campo, que según Sandoval (1997) es una herramienta fundamental en la etnografía, anotando así todas las observaciones realizadas y posteriormente interpretadas. La observación fue de tipo participante, lo que, al decir de Denzin (1978, citado en Ruiz, 2003) "es una estrategia de investigación en la que el observa-

3 Entiéndase no-torneos como momentos en que se juega de manera consensuada según las disposiciones de tiempo, de lugar y jugadores sin que haya institucionalización o trofeo oficial. dor tiene un papel activo" (p. 27), esto porque dicho observador también se hizo partícipe de los partidos de microfútbol (en este caso, no-torneos) antes y durante la investigación. También se realizaron entrevistas semiestructuradas (Ander-Egg, 1995), en las cuales aunque se lleva un guion temático, se pueden tratar y discutir temas emergentes propuestos por el entrevistado; al mismo tiempo se dieron conversaciones sin una previa planeación. Por último, se hizo una revisión documental detallada a partir de grabaciones de audio realizadas mientras se daban las entrevistas para no omitir datos que pudieran ser muy relevantes, lo anterior con el fin de contribuir a la comprensión de los aportes que hacen los participantes y sujetos implicados de los campos de juego con una mayor profundidad.

\section{RESULTADOS Y DESARROLLO DEL TRABAJO DE CAMPO}

\section{La ley del más fuerte}

La cancha de microfútbol se puede entender como un campo (en este caso de juego), es decir como aquel espacio social dinámico y estructurado donde existen jerarquías, una red de relaciones y reglas de juego propias (Bourdieu, 2000).

Con base en este planteamiento, y a partir de las mencionadas observaciones, se pudieron analizar las relaciones estructurales basadas en la ley del más fuerte, en la que se propone una jerarquía. A manera de relatoría, al llegar a la primera cancha se observó que había dos equipos de niños jugando, cuyas edades probablemente oscilaban entre los 9 y los 14 años; pasados 15 minutos, llegó un grupo de jóvenes ya en una edad adulta, cuyas edades podrían oscilar entre los 17 y los 27 años. Cuando ellos entraron a la cancha y empezaron a jugar, los niños dejaron hacerlo y se hicieron a un lado, como sabiendo ya lo que deben hacer cuando llegan grupos de mayores.

En una nota realizada por la emisora Caracol Radio, en la que se destacan los cinco deportes ${ }^{4}$ más ejecutados en Bogotá (2017), se nombran, en su orden, del menos al más ejecutado: atletismo, bicicrós, pati-

4 Entiéndase deporte como una actividad institucionalizada, competitiva y reglada (Parlebas, 1993) 
naje, fútbol y microfútbol. Aunque la nota no contiene ningún soporte de porcentajes o fuentes, puede pensarse en que tienen algo de veracidad (al menos en el microfútbol), pues en el barrio es difícil encontrar un parque sin una cancha de dicho deporte, o donde la sola cancha sea el parque en sí mismo. De allí la relevancia que le dan las entidades recreo-deportivas a dicho deporte.

Y es que el auge, la fama y la importancia del microfútbol para las comunidades, en especial niños y jóvenes, parece ser más que evidente. Por ejemplo, los niños sacados de la cancha principal -mencionados anteriormente- tomaron unas cuantas piedras e hicieron sus propias canchas en lugares dispuestos para que las personas caminen por el parque, e incluso otros niños y jóvenes ya están dentro de la cancha de baloncesto jugando sus partidos de microfútbol también en canchas improvisadas, estorbando hasta cierto punto a los jugadores de baloncesto, lo que desata algunas discusiones acaloradas. Es allí donde el microfútbol comienza a dar las primeras luces de ser una actividad no tan constructora de tejido social (recreativa), en la que, si bien es cierto se pueden dar relaciones de amistad, no germina allí, sino que puede configurar una parte más oscura de dicho juego.

\section{Programas recreativos para la convivencia en el sector}

El segundo día del proceso observacional (sábado), nuevamente se hizo un acercamiento a la cancha 1 , a eso de las 8:30 a. m.; allí se encontraba alguien que parecía ser un padre jugando con sus dos hijos. Llegadas las 9:00 a. m. al lugar se asomó un grupo de personas con mallas para las canchas, al tiempo que equipos de fútbol comenzaron a darse al encuentro, por lo cual el padre se debió retirar del lugar de juego con sus hijos.

Al realizarse un acercamiento a los que estaban dirigiendo la actividad, se inició una conversación en la cual el observador hizo las siguientes preguntas de manera espontánea:

Observador: Buenos días. Una pregunta: ¿de qué hora a qué hora están aquí jugando partidos?
Organizadora: El torneo va de 9 (a. m.) a 9 (p. m.), los sábados y domingos.

Observador: ¿Todo el año?

Organizadora: Sí

De allí nace entonces una primera apreciación, y es que estas canchas ya tienen un uso dispuesto para la realización de torneos oficiales casi la mayoría de horas seguras en las que un padre puede salir a jugar con sus hijos (las horas diurnas), teniendo en cuenta también que estos torneos se realizan en los días que son comúnmente de descanso (sábados y domingos) para los adultos, es decir, probablemente los únicos en los que dicho padre podría dedicar tiempo para jugar con sus hijos. Lo mismo podría suceder con aquellos jóvenes y niños que, a causa de sus deberes académicos y del hogar, o por la inseguridad del sector, solo tienen tiempo o se les permite salir los fines de semana, que es cuando más afluencia de personas hay en las calles.

Dando continuidad a la conversación, el observador pregunta: “ ¿Y por qué se están haciendo estos torneos?", a lo que la organizadora responde: "Somos los organizadores de un proyecto de la Alcaldía Local para la mejora de la convivencia y la tolerancia en muchachos del sector a través de actividades recreativas, y pues también alejarlos un poco de las drogas, también estamos dando teatro y Break Dance por si quieres inscribirte". Y es allí donde se ve la importancia de la conceptualización, incluso a escala gubernamental, de qué se entiende por recreación y hacia qué va dirigida, así como la divulgación a los representantes de dichos proyectos dentro de la población. Es claro que ni siquiera se hace una diferenciación entre lo deportivo y lo recreativo.

Al observarse algunos partidos posteriores a la charla con la organizadora, se presenta una serie de sucesos. En el primero de ellos, uno de los jóvenes comete falta y el otro le responde con una grosería, incluso algunos espectadores (posiblemente conocidos, amigos o familiares del que recibió la agresión en primer lugar) hacen lo mismo contra el jugador que cometió la falta. Algo similar ocurre en otro partido, en el cual se da una discusión originada por los roces 
del partido, y algunos jugadores suplentes entran al campo de juego al parecer con intenciones de pelear.

Ahora, tal y como menciona Pedraza (1997):

El deporte ha sido mostrado y [...] utilizado como la enmienda para los transgresores de la ley de la normalidad: moral para amorales, correctivo para delincuentes, revulsivo para vagos, purga para drogadictos, templanza para violentos, pedagogía para inadaptados, ilustración para ignorantes, esperanza para desahuciados, etc. (p. 45).

Sin embargo, como se ha mencionado, es el uso indiscriminado del lenguaje entre lo recreativo y lo deportivo, así como la perspectiva de ver estos conceptos como generadores de un estado de paz, amor, tranquilidad y convivencia. Esto no permite hacer entender que dentro de estas dinámicas se producen factores tales como la violencia, la agresividad, el irrespeto y todas aquellas cosas que pueden ser el opuesto de lo que llamamos valores o moral. Esta desinformación lleva a un imaginario utópico de dichos conceptos y saberes en cuanto a la recreación o el deporte, lo que impide reconocer y trabajar en sus problemáticas, que pueden repercutir en escenarios incluso peores para una sociedad.

\section{Un lenguaje al reto y a la amabilidad: "Echar} la luca" y "juegue suave que es de recocha"

Siendo miércoles en la noche, y aunque no muy seguro, se continuó con el proceso observacional en la cancha 2 a las 9 p. m. En esta ocasión, el observador acompañó a un grupo de amigos a jugar (intervino en el partido), y llevó al lugar una serie de preguntas (la entrevista semiestructurada) para entrevistar a algún jugador del equipo contrario. Como solo había un grupo de tres personas contra las cuales jugar en el parque, fue necesario esperar a que llegaran otros participantes; tiempo después llegaron tres más, y de ellos dos jugaron (el otro se quedó sentado porque el equipo ya estaba completo). "Ya está el combo", mencionó uno de los participantes, haciendo alusión a que ya estaba listo el equipo que iba a jugar. "Vamos a echar la luca", dijo la misma persona, refiriéndose a apostar mil pesos, lo que parece emocionar a algunos y poner algo de tensión en el juego tal como lo describe Huizinga (1938).
Inicialmente se dio un partido tranquilo, pero con el paso del tiempo y de goles se caldearon los ánimos debido al sentido de competencia creado por el deseo de ganar los mil pesos. Como lo describe Caillois (1986) en cuanto al Agon (competencia), es una subcategoría del juego en donde hay un enfrentamiento entre antagonistas y se da una lucha en una igualdad artificialmente creada. Algunas condiciones de la competencia y de la necesidad de ganar se manifestaban con más fuerza cuando, aparte de los roces entre jugadores de equipos contrarios, se daban roces entre jugadores del mismo equipo con frases como "no sea bobo ¿cómo va a botar ese gol?" o "salgase mejor si va a seguir jugando así".

Se reflexiona, entonces, en que efectivamente la competitividad puede llegar a ser un proceso riesgoso, si se vuelve una hipercompetitividad (competencia llevada a niveles demasiado altos), sobre todo al hablar de zonas tan susceptibles como el barrio y la localidad, en temas de violencia y conflicto interno. Este fenómeno se evidenció en las dos canchas, tanto en el torneo como en el partido donde se apostó dinero. Incluso, una discusión en un partido llegó a provocar disparos y un herido (situación ya antes vivida en una de las canchas durante otros torneos).

Al terminarse el partido, un equipo manifestó que quería jugar pero no tenía dinero, así que quería que jugaran de "recocha", es decir, sin apostar dinero, ni añadir algún otro valor o sentido más allá del de jugar. A pesar de esto, en el partido hubo tensión y algo de competencia; un hecho interesante se presentó cuando uno de los jugadores tuvo un contacto físico un tanto fuerte y vigoroso con un jugador del equipo contrario, razón por la que este cayó, pero al levantarse le dijo "Suave, parcero, que estamos jugando de recocha”. Los demás jugadores, incluso los del equipo del que cometió la falta, parecieron estar de acuerdo con estas palabras y, probablemente por esta razón, el contacto físico a lo largo del partido se dio de manera más leve. En este caso es notable y se destaca el poder de autorregulación y la competitividad sin llegar a la hipercompetitividad cuando no hay un fin más allá del juego y el ganar (sin que necesariamente esto sea lo más importante), es decir, cuando se pueden eliminar los trofeos y las medallas o las recompensas demasiado valiosas, y el jugador 
se puede entregar al juego por el disfrute de jugar y de sentirse pleno.

Es de tal magnitud lo mencionado, que dentro de dicho juego de "recocha" se les permitió jugar a los jugadores que los mismos participantes consideraban "malos", sin hacerles ningún reclamo por perder un partido. Es entonces cuando la importancia del juego como verdadera y auténtica manifestación recreativa aparece en escena como constructor del tejido social y el proceso incluyente que permite la aceptación de las personas sin importar sus habilidades o capacidades.

\section{De la violencia y la necesidad de la recreación: "Es peligroso, pero vale la pena"}

Al terminarse el partido el observador se acerca a uno de los jugadores del equipo contrario, y le pide su colaboración para entrevistarlo. El joven dice llamarse Juan Pablo (JP), y entre sus respuestas comenta que vive allí desde que nació (22 años).

Algunas de las preguntas a las que se les dio más relevancia fueron si no le daba miedo salir a jugar tan tarde, a lo que respondió: "Es peligroso, pero vale la pena, porque a veces uno llega estresado del trabajo, entonces saliendo con los amigos uno se relaja un poco, igual nacimos para morirnos". Dice que trabaja en construcción, vive con su mamá y su hermana, porque su papá los abandonó cuando él tenía cuatro años, y que le toca pagar la mayoría de gastos en su hogar.

Si bien es cierto que dichas actividades recreo-deportivas o de ocio no se podrían ver como una panacea (en este caso el microfútbol), es evidente que hacen las veces de desfogue en las personas para el descanso, la diversión y el desarrollo de las personas, tal como afirma Dumazedier (1971). Y en este caso podría hacer las veces de una de las reducidas posibilidades de recreación que tiene JP, ya que menciona "todo lo que me gano es para la casa y no me queda casi para nada más, ni viajar, ni salir y como tengo a mi mamá y a mi hermana, conseguir novia en estos momentos, no puedo, porque ni tiempo me queda porque tengo que cuidar a mi mamá que no puede ya trabajar".

No solo queda destacar en este caso la importancia de las actividades recreativas para las personas en cuanto a escapatoria de su diario vivir, incluso evidenciar el valor de dichos jóvenes para salir a jugar a pesar del peligro de que en cualquier momento puedan llegar grupos armados de limpieza social (asesinos que aseguran eliminar personas que le hacen daño a la sociedad) y asesinarlos, como ha ocurrido tantas veces en el sector. Esto permite apreciar la importancia que le dan estos habitantes al hecho de poder salir a jugar para olvidar o alejarse un poco de los problemas del diario vivir en una cotidianidad dada por sus factores de entorno, entre ellos violencia, trabajo, pobreza y necesidad, así como el sueño de que en algún momento su situación cambie.

\section{CONCLUSIONES}

Como producto de esta investigación queda el observar, entre otras problemáticas, la falta de conceptualización y divulgación, así como la tergiversación del concepto de recreación en las comunidades (por ejemplo lo recreo-deportivo). Una posible razón es el desinterés hacia dicho campo, ya que la recreación podría verse en niveles estatales- gubernamentales, como algo en que las personas van a divertirse y pasarla bien, así como a descansar para después rendir mejor en sus actividades de trabajo o estudio.

La recreación, en este caso desde el microfútbol, queda a merced de no tocar problemáticas que esta genera, tales como la violencia, la inseguridad, la no tolerancia, el quitar espacios de juego para la familia, e incluso llegar a topes en que genera la destrucción del tejido social (algo por lo cual el concepto se debe seguir debatiendo), sobre todo en espacios llamados recreo-deportivos, en los cuales se llega a generar la hipercompetitividad.

En relación con la hipercompetitividad, se podrían generar espacios en los cuales, aunque haya una mediación, se podrían quitar factores como el premio, para generar otros espacios (mundos posibles) en cuanto a la formulación de torneos en microfútbol.

\section{REFERENCIAS}

Alcaldía Local de Ciudad Bolívar. (2016). Conociendo mi localidad. Recuperado de http://www.ciudadbolivar. gov.co/mi-localidad/conociendo-mi-localidad/historia.

Ander-Egg (1995). Aprender a investigar: Nociones básicas para la investigación socialCórdoba: Brujas. 
Bourdieu, P. (2000). Las formas del capital. Capital económico, capital cultural y capital social. En Poder, derecho y clases sociales. Bilbao: Desclée de Brouwer.

Caillois, R. (1986). Los juegos y los hombres, la máscara y el vértigo. México: FCE.

Caracol Radio. (2017, 27 de febrero). Los 5 deportes más practicados en Bogotá. Recuperado de http://caracol.com.co/emisora/2017/02/27/ bogota/1488195991_095382.html.

Dumazedier, J. et al. (1971). Ocio y sociedad de clases. Barcelona: Fontanella.

Fetterman, D. M. (1989). Ethnography step by step. Beverly Hills-California: Sage.

Huizinga, J. (1972). Homo ludens. Madrid: Alianza Editorial.

Murillo, F. J. y Martínez-Garrido, C. (2010). Investigación etnográfica. Madrid: UAM.

Moreno, A. C. y Moreno, J. (2011). Ciudad Bolívar: diferencias culturales y políticas en contacto. Una mirada sociolingüística a la migración y el desplazamiento forzado en Colombia (p. 58). Recuperado de http://biblioteca-
digital.caroycuervo.gov.co/189/1/02_Ana_Constanza_ Rodriguez_Javier_Moreno_Valero_0.pdf .

Parlebas, P. (1993). Problemas del juego en la educación física. En Actas Primer Congreso Argentino de Educación Física y Ciencias. Departamento de Educación Física, FHCE-UNLP, La Plata.

Pedraza M. V. (1997). Poder y cuerpo. El (incontestable) mito de la relación entre ejercicio físico y salud. Educación Física y Ciencia, 3, 7-19.

Ruiz Olabuenaga, J. I. (2003). Metodología de la investigación cualitativa. Bilbao: Universidad de Deusto.

Sandoval (1997). Investigación cualitativa. Colombia: ARFO Editores e Impresores.

Universidad Pedagógica Nacional. (2016). Documento maestro. Licenciatura en Recreación y Turismo, p. 36.

Universidad del Rosario. (2009). Universidad, ciencia y desarrollo. Recuperado de http://www.urosario.edu.co/Universidad-Ciencia-Desarrollo/ur/ Fasciculos-Anteriores/Tomo-IV-2009/Fasciculo Localidad-de-Ciudad-Bolivar/--3/ur/. 